\title{
Application of Treatment of Sanyinjiao in Gynecological Diseases Based on the Relevance of Zang-fu Meridians and Collaterals
}

\author{
Wang Aijing ${ }^{1}$, He Fengjie ${ }^{2}$ \\ ${ }^{1} 2018$ postgraduate students in Shanxi University of Chinese Medicine, Xianyang Shanxi 712000 \\ ${ }^{2}$ Affiliated Hospital of Shanxi University of Chinese Medicine, Xianyang Shanxi 712000
}

How to cite thi paper: Wang, A., He, F. (2019) Application of Treatment of Sanyinjiao in Gynecological Diseases Based on the Relevance of Zang-fu Meridians and Collaterals. International Journal of Clinical and Experimental Medicine Research, 3(4), 131-134.

DOI: 10.26855/ijcemr.2019.10.004

Corresponding author: Wang Aijing, 2018 postgraduate students in Shanxi University of Chinese Medicine, Xianyang Shanxi 712000.

Email: 3467322995@qq.com

\begin{abstract}
Sanyinjiao is the intersection point of spleen meridian, liver meridian and kidney meridian.And it is closely related with vital channel, ren channel, uterus and internal organs. Sanyinjiao has the function of regulate human health, and has remarkable treatment effection on female diseases.

Sanyinjiao, first recorded in "Acupuncture and moxibustion" [1], is located 3 inches above the tip of the medial malleolus and at the posterior margin of the medial side of the tibia [2]. Sanyinjiao acupoint is named Sanyinjiao because of the damp and hot Qi provided by the spleen, the water and wind provided by the liver and the cold Qi provided by the kidney. Kidney stores essence, which is the source of "Tiangui"; spleen controls blood, which is the source of blood biochemistry; liver stores blood, which is the source of meridians. The growth and development of women can not be separated from the participation and regulation of liver, spleen and kidney. Sanyinjiao, as the intersection point of foot three yin meridians, is closely related to viscera, meridians and uterus, which determines its particularity and importance in gynecology physiology and pathology. Through acupuncture at Sanyinjiao single point and its compatibility, it can regulate viscera in two ways, improve viscera function, and regulate Chongren uterus. The occurrence of common gynecological diseases in clinic is mostly in the uterus, and the changes are mostly in the viscera and Qi and blood. Most of the causes are caused by the loss of liver and kidney or the injury of Chong Ren. Because Sanyinjiao is closely related to the viscera, meridians and the uterus, Sanyinjiao point is the first choice for the treatment of gynecological related diseases. The author summarizes the physiological connection, pathological diseases and treatment of Sanyinjiao in gynecology as follows for reference.
\end{abstract}

\section{Keywords}

\section{The relationship between Sanyinjiao and the uterus}

The uterus, also known as the womb, uterus, and inner organs. It is located in the middle of the abdomen, under the vein, with bladder in front, rectum in the back and vagina in the lower part. According to Fu Qingzhu's theory, "the fetus is unique outside the five zang organs, and its nature is not cloudy, not cold, not hot, and is adaptable to change.". As the organs of Qi Heng, the uterus has the characteristics of both storing and discharging, and storing and writing regularly. 
Its main function is to excrete menstruation and to gestate the fetus. When the blood of Yin overflows in the pulse, the blood of Yin accumulates in the Ren pulse and the blood of Yin maintains in the Du pulse, the uterus can come on time and the woman can gestate the fetus.

"One Qi and three sources" of Chong, Ren and Du Meridians originated from the uterus, closely related to the foot three yin meridians. Ren Mai is in charge of the Yin of the whole body, and all three yin channels can be found in Ren Mai. When viscera, Qi and blood, Chong Ren and other diseases indirectly damage the uterus, or the uterus is directly damaged, it can lead to the occurrence of gynecological diseases. By acupuncture at Sanyinjiao point, the viscera, Qi and blood, Chong Ren can be adjusted, and the uterus can recover its normal physiological function, so as to treat gynecological diseases.

\section{The relationship between Sanyinjiao and viscera}

\subsection{The relationship between Sanyinjiao and liver}

The liver stores blood, which is the main part of catharsis. It has the functions of storing blood, regulating blood volume and catharsis mechanism. It is an important link of regulating the whole body Qi mechanism and promoting blood and body fluid operation. Women are born with liver and blood as the foundation; the two blood vessels of Chong Ren are connected with liver, and the liver and Chong pulse intersect at Sanyinjiao, and the two blood vessels of Ren and Du act on the uterus together. When the function of regulating and relieving liver Qi is normal, the Qi and blood of the whole body is usually peaceful, and the menstruation, belt, fetus and labor of women can be carried out normally and orderly. Women's blood is less and Qi is more. When the liver is out of place and Qi and blood is out of balance, it is easy to cause diseases such as meridians, belts, foetuses and childbirth. Acupuncture at Sanyinjiao can calm the liver Yang, nourish blood and soften the liver, and dredge the liver qi.

\subsection{The relationship between Sanyinjiao and spleen and stomach}

Spleen and stomach are the foundation of postnatal life and the source of blood biochemistry. They can transport and transform water valley and generate Qi and blood, providing material basis for women's menstruation, belt, fetus and birth. The spleen governs the blood collection, the stomach governs the reception, and the spleen and stomach work together to transport the essence of shuigu to the whole body; when shuigu is flushed in the stomach, the blood of chongmai will be flushed, and menstruation will come on time. If the spleen and stomach are healthy, the Qi will be absorbed and the blood will be absorbed, the blood will be mixed, the menstruation will come, the uterus will be nourishing, and the fetus will be born. If the spleen and stomach are weak, the circulation of Qi and blood is disordered, the biochemical source of Qi and blood is deficient, and the Qi and blood of the human body are deficient, or the Qi and blood are deficient and unable to control the blood, so that the blood does not follow the meridians, it is easy to lead to the occurrence or aggravation of diseases such as meridians, belts, foetuses, births, and breasts. Sanyinjiao is the spleen meridians of foot Taiyin. The circulation of the spleen meridians "goes into the abdomen, which belongs to the spleen and collaterals the stomach". Sanyinjiao has the functions of promoting blood circulation and regulating the meridians, strengthening the spleen and promoting dampness, and regulating the spleen and stomach. Therefore, the spleen and stomach can be transported by Acupuncture Sanyinjiao, and the Qi and blood are vigorous, so the disease can be eliminated by itself.

\subsection{The relationship between Sanyinjiao and kidney}

The kidney stores essence and is mainly for reproduction. The connection with the uterus is the basis of human growth, development and reproduction, and directly provides material basis for the menstruation and pregnancy of the uterus. Kidney qi Chong Sheng promoted Tiangui to mature, through Ren and Chong two vessels acting on the uterus, in order to complete the reproductive development function. Whether the menstruation is normal or not, whether the reproductive ability is strong or not, depends on the rise and fall of kidney qi. If congenital deficiency of kidney qi or acquired damage of kidney qi, or atrial labor and Prolificacy, or long-term serious disease, will lead to the occurrence of related gynecological diseases. Although Sanyinjiao acupoint is not a kidney meridian acupoint, the circulation of kidney meridian passes through Sanyinjiao, Sanyinjiao passes through foot Shaoyin kidney meridian, foot Shaoyin kidney meridian also strengthens the communication and contact of Ren vessel and uterus through Sanyinjiao. Acupuncture at Sanyinjiao can supplement essence, generate blood, warm and moisten essence, balance yin and Yang, so as to make kidney qi flush. 


\section{Treatment of diseases by Sanyinjiao}

\subsection{Treatment of menorrhea by Sanyinjiao}

The disease location of menstrual disease is mostly in the uterus, which is often caused by external pathogenic factors, emotions, life, constitution and other factors, resulting in liver, spleen, kidney and viscera disorders, indirect or direct damage to Chong, Ren, Du, Dai and the uterus, resulting in Chong and Ren disorders, dysfunction of viscera and Qi blood disharmony. Therefore, the treatment of menstrual diseases starts from three aspects: regulating qi and blood, tonifying the viscera, and regulating and strengthening the Chong Ren. (1) harmonizing Qi and blood: for menstrual advance, excessive menstrual volume, prolonged menstrual period and metrorrhagia caused by deficiency of Qi and blood, Sanyinjiao, Qihai and Guanyuan can be selected as the main acupoints to regulate qi and blood, consolidate the intake of Chong Ren, consolidate collapse and stop bleeding. Sanyinjiao can regulate the liver, spleen and kidney, and has the effect of nourishing qi and blood; Guanyuan is the source of Sanjiao Yuanqi, which is Mingmen Zhenyang, which is the Yang point in Yin, which can nourish the liver and kidney; Qihai is the sea of Yuanqi, which is the whole body of Yuanqi, which can nourish Qi and blood. In the late period of menstruation, too little menstrual volume, dysmenorrhea and amenorrhea caused by stagnation of liver qi and cold coagulation and blood stasis, the main points are Sanyinjiao, Hegu, Taichong and Zhongji, which can activate Qi and blood circulation, remove blood stasis and dredge collaterals, and relieve pain of dysmenorrhea. Sanyinjiao is matched with Hegu. It can nourish Hegu and reduce Sanyinjiao. It has the function of promoting qi and blood circulation, clearing channels and removing blood stasis. Taichong can dredge Qi and relieve depression, regulate blood and regulate channels. Zhongji is renmai acupoint, which can regulate qi and blood, regulate the Qi of focusing, and play the role of near treatment of "acupoint, main treatment". (2) tonifying the viscera: mainly tonifying the kidney, strengthening the spleen and liver. Sanyinjiao is the intersection point of foot, Sanyin and meridians, which is based on nourishing the liver, spleen and kidney. It is combined with Mingmen, Guanyuan, Shenshu, warming the kidney yang, tonifying the kidney qi and warming the uterus. It is used to treat the deficiency of kidney qi and kidney yang and the later period of menstruation. Combined with Zusanli, Pishu and Weishu, it can strengthen the spleen and stomach, strengthen the body and Qi, and treat menorrhea and metrorrhagia. With Taichong, Jimen and Taixi, it can treat dysmenorrhea and menorrhea with stagnation of liver qi and deficiency of liver blood. (3) regulating and strengthening Chong Ren: when Chong Ren's deficiency and loss, and Gu Shi's dereliction of duty, metrorrhagia, menstrual disorder and prolonged menstrual period may occur. Sanyinjiao can be selected to consolidate Chong Ren, control blood, plus renmai acupoints, Guanyuan and Qihai to strengthen the function of consolidating Chong Ren.

\subsection{Sanyinjiao in the treatment of diseases under the belt}

The occurrence of the disease is often related to the feeling of dampness, physical weakness, food fatigue and other factors. The location of the disease is mostly in the uterus, which is closely related to the meridians, Ren vessels, spleen and kidney. The etiology of the disease is nothing more than dampness. Dampness leads to the unsteadiness of the Ren and the incoordination of the Qi, which leads to the abnormality of the Qi. Treatment of serious diseases under the belt in the spleen dehumidification, Yang stop. Take Sanyinjiao, Zhongji, daimai and baihuanshu as the main points, Sanyinjiao as the point of spleen meridian, eliminating dampness and stopping band, to cure its root; Zhongji is the intersection point of Ren meridian and foot Sanyinjiao, which has the function of consolidating dampness, strengthening the spleen and benefiting the kidney; daimai is the place where meridians pass, which can adjust Chong Ren, stopping band, regulating meridians blood and regulating Sanjiao; baihuanshu is the point of foot Taiyang meridian, which can adjust bladder gasification and promoting dampness and stopping band.

\subsection{Sanyinjiao in the treatment of delayed labor}

As for the occurrence of dystocia, the disease is located in the uterus, and is closely related to Chong, Ren and kidney. In terms of etiology and pathogenesis, it is mainly caused by Qi and blood disorders; those with weak Qi and blood are sluggish due to inability to transport the fetus, and those with stagnation of Qi and blood are sluggish due to stagnation of the fetus. As for acupuncture treatment of delayed childbirth, "dystocia: Hegu (tonic), Sanyinjiao (purgative) and Taichong" is proposed in Dacheng women's gate of acupuncture [3] in terms of treatment, the principle of treatment is to regulate qi and blood, and to induce labor with stagnation. The main points are Sanyinjiao, Hegu, Zhiyin and Jianjing. Hegu belongs to the large intestine meridian of hand Yangming, which is the original point of the large intestine meridian. The lung and the large intestine are in the exterior and in the interior, and the lung is in the main. Therefore, the method of acupuncture Hegu can make the fetal Qi move in a false way. Sanyinjiao is the main blood, 
acupuncture can activate blood and blood by catharsis, and the matching of the two can activate blood and Qi and give birth. Zhiyin is the well point of the foot sun meridian, which can benefit the kidney qi and regulate the uterus; Jianjing is the intersection point of foot Shaoyang meridian, Foot Yangming and Yang Weimai, which can benefit the Yang Qi and help the fetus to descend. Zhiyin and Jianjing are both the experience points of induced abortion.

\subsection{Sanyinjiao for postpartum lochia}

The clinical manifestations of postpartum lochia are mostly postpartum blood lochia for more than 10 days, or have a stench, which can be accompanied by mental fatigue, shortness of breath, sloth, hollow abdomen, or refusal to press with pain in the abdomen. Anemia can be combined with bleeding for a long time, and syncope can be caused in severe cases. The evil dew comes from the palace and is injected into Chong Ren. It is turned into blood. Therefore, for the treatment of lochia, the focus is to harmonize qi and blood, consolidate the intake of Chong Ren, differentiate the quantity, color, quality and taste of lochia, and combine with the general symptoms of parturient women. The main acupoints of this disease are Sanyinjiao, Guanyuan, Qihai and Xuehai. Sanyinjiao is an important point for regulating blood circulation and regulating meridians, which has the function of invigorating the uterus and strengthening the spleen and regulating blood; Qihai and Guanyuan are renmai points, which are close to the uterus and intersect with foot Sanyinjiao, which can benefit the vitality, strengthen the Chong and Ren, and regulate the uterus, so that the external meridians of the uterus can return to the meridians; Xuehai can activate Qi and blood, and strengthen the curative effect. According to the syndrome differentiation of Linchuan, those with deficiency of Qi should be matched with Zusanli and Pishu, invigorating the spleen and benefiting qi to control blood, blood heat should be matched with middle pole and line, cooling blood and removing blood stasis to stop bleeding, qi stagnation should be matched with Geshu and Diji, activating blood and removing blood stasis to dredge collaterals. "Acupuncture integration" records: "postpartum lochia not only, Yin cross hundred strong."

\section{Summary}

Sanyinjiao, as a common point of gynecological diseases, has the functions of Tonifying the liver and kidney, regulating Chong Ren, nourishing the uterus, etc. it is not only a common point for the treatment of women's menstruation, belt, fetus and childbirth, but also known to the public. Regular massage has a health care effect. In the face of complex clinical gynecological diseases, it is necessary to combine the patients' systemic symptoms, four diagnosis and careful syndrome differentiation, in order to achieve accurate and effective treatment.

\section{Reference}

[1] edited by Yang Jizhou, edited by Jin Xian, edited by Huang Longxiang. Acupuncture Dacheng [M]. Beijing: People's Health Press, 2006: 1-492

[2] Shi Xuemin. Acupuncture [M]. Beijing: China traditional Chinese Medicine Press, 2007: 49

[3] Yu Bing, Wang Cong, Zhang Yongchen. Analysis on the clinical application of Sanyinjiao point in acupuncture Dacheng [J]. Sichuan Traditional Chinese medicine, 2016,34 (07): 1-3 